\title{
Investigation of Antiphase Domain Boundaries in Cobalt Ferrite Thin Films via High Resolution Scanning Transmission Electron Microscopy
}

Amanda Trout ${ }^{1}$, Igor Pinchuk ${ }^{2}$, Michael Newburger ${ }^{2}$, Roland Kawakami ${ }^{2}$ and David McComb ${ }^{1,3}$

${ }^{1}$ Center for Electron Microscopy and Analysis, The Ohio State University, Columbus, Ohio, United States, ${ }^{2}$ The Ohio State University, Columbus, Ohio, United States, ${ }^{3}$ Department of Material Science and Engineering, The Ohio State University, Columbus, Ohio, United States

Cobalt ferrite is an inverse spinel ferromagnetic insulator that has been shown to induce magnetism in platinum through a phenomenon called magnetic proximity effect. ${ }^{1}$ Complete understanding of the spin manipulation at the interface of cobalt ferrite and platinum has been hindered due to the complex nature of the cobalt ferrite structure. Cobalt ferrite is an inverse spinel of space group Fd-3m. The unit cell consists of $\mathrm{O}$ ions in an FCC lattice with $1 / 8$ of the tetrahedral sites occupied by $\mathrm{Fe}^{3+}$ ions and $1 / 2$ of the octahedral sites occupied by $\mathrm{Co}^{2+}$ and $\mathrm{Fe}^{3+}$ ions. The fraction of octahedral sites occupied by trivalent atoms is known as the degree of inversion $(\chi)$, and the reported value is $\sim 0.75-0.8$ for cobalt ferrite. ${ }^{2}$

Complete characterization of the cobalt ferrite structure is needed in order to understand its magnetic properties and the spin manipulation that occurs at the $\mathrm{CoFe}_{2} \mathrm{O}_{4}$ /platinum interface. We have used high resolution STEM imaging and analytical techniques to investigate cobalt ferrite thin films grown on $\mathrm{MgO}$ via molecular beam epitaxy. Energy dispersive X-ray spectroscopy indicates that the thin film is homogeneous with a sharp interface between the $\mathrm{CoFe}_{2} \mathrm{O}_{4}$ and $\mathrm{MgO}$. The cross sectional HAADF image (Figure 1) shows the atomic interface with a cube on cube epitaxial relationship and the expected spinel structure. However, there is also a variation in the image contrast throughout the thin film causing some regions to appear cubic.

It has been shown that magnetite $\left(\mathrm{Fe}_{3} \mathrm{O}_{4}\right.$, spinel structure) thin films contain antiphase boundaries caused by a shift along a/4[110], which can been seen when imaged with a (220) beam. ${ }^{3,4}$ We believe that the contrast variation in our experimental HAADF images may also be due to the presence of antiphase domains in our cobalt ferrite thin films. $\square$ STEM $^{5}$ was used to simulate HAADF images of a cobalt ferrite structure that contains an antiphase boundary along 1/4[110]. The simulated images show the spinel structure and a variation in the image contrast similar to our experimental HAADF images with regions that appear cubic. We also used high resolution TEM imaging along [001] to collect images of the antiphase domains present in our cobalt ferrite thin films. Figure 2 shows several antiphase boundaries on the order of $5-10 \mathrm{~nm}$.

In this contribution, we will further discuss using simulated and experimental S/TEM imaging and analytical techniques to investigate the presence of antiphase domains in cobalt ferrite thin films. We are exploring the use of Lorentz microscopy to study the effects of the antiphase domains on the magnetic domains in $\mathrm{CoFe}_{2} \mathrm{O}_{4}$. We are also exploring the use of integrated differential phase contrast (iDPC) in combination with electron energy-loss spectroscopy to further characterize the antiphase domains present in our $\mathrm{CoFe}_{2} \mathrm{O}_{4}$ thin films. 


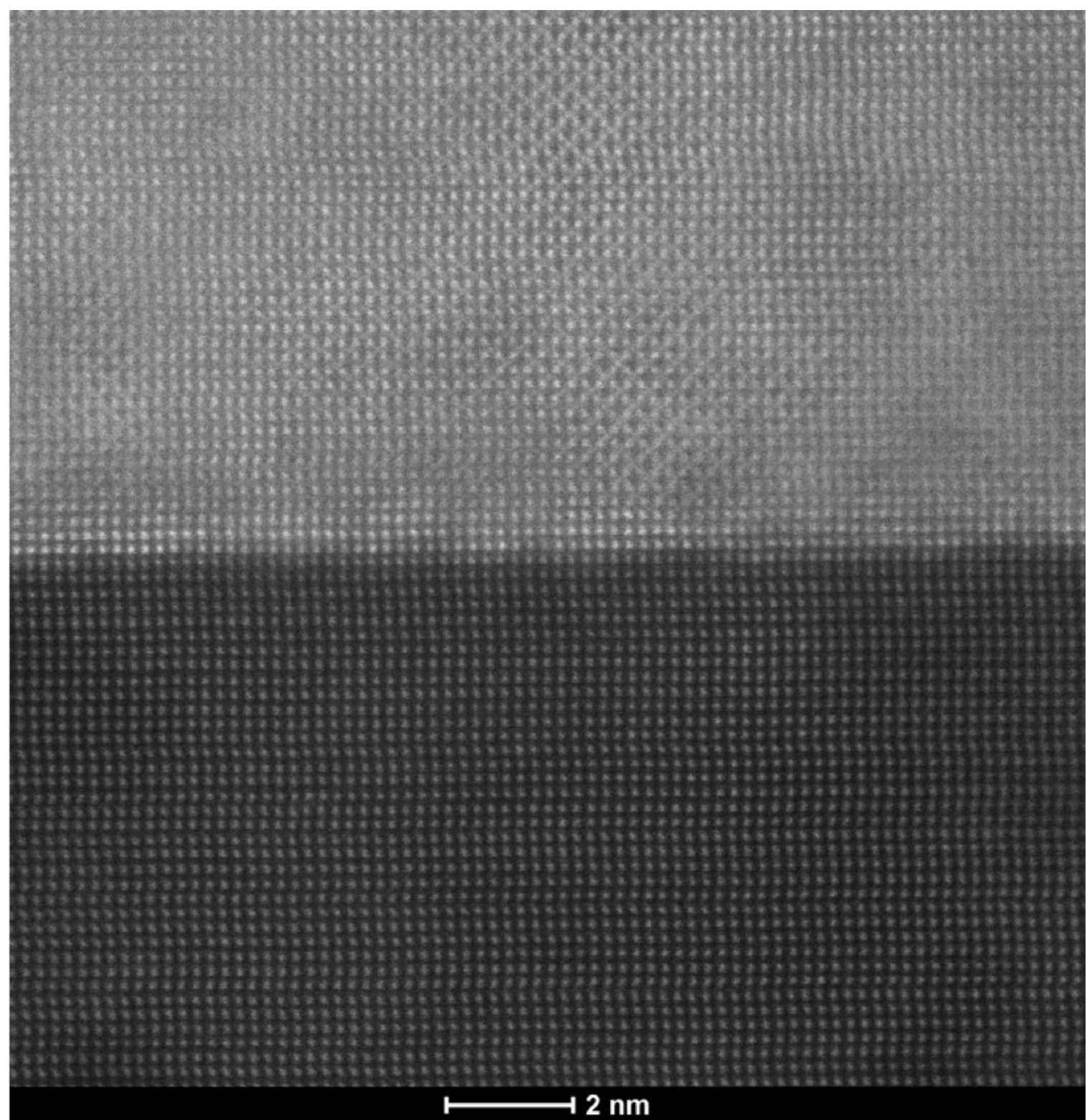

Figure 1. Cross-sectional HAADF image showing the $\mathrm{CFO} / \mathrm{MgO}$ interface, the spinel structure, and contrast variation. 


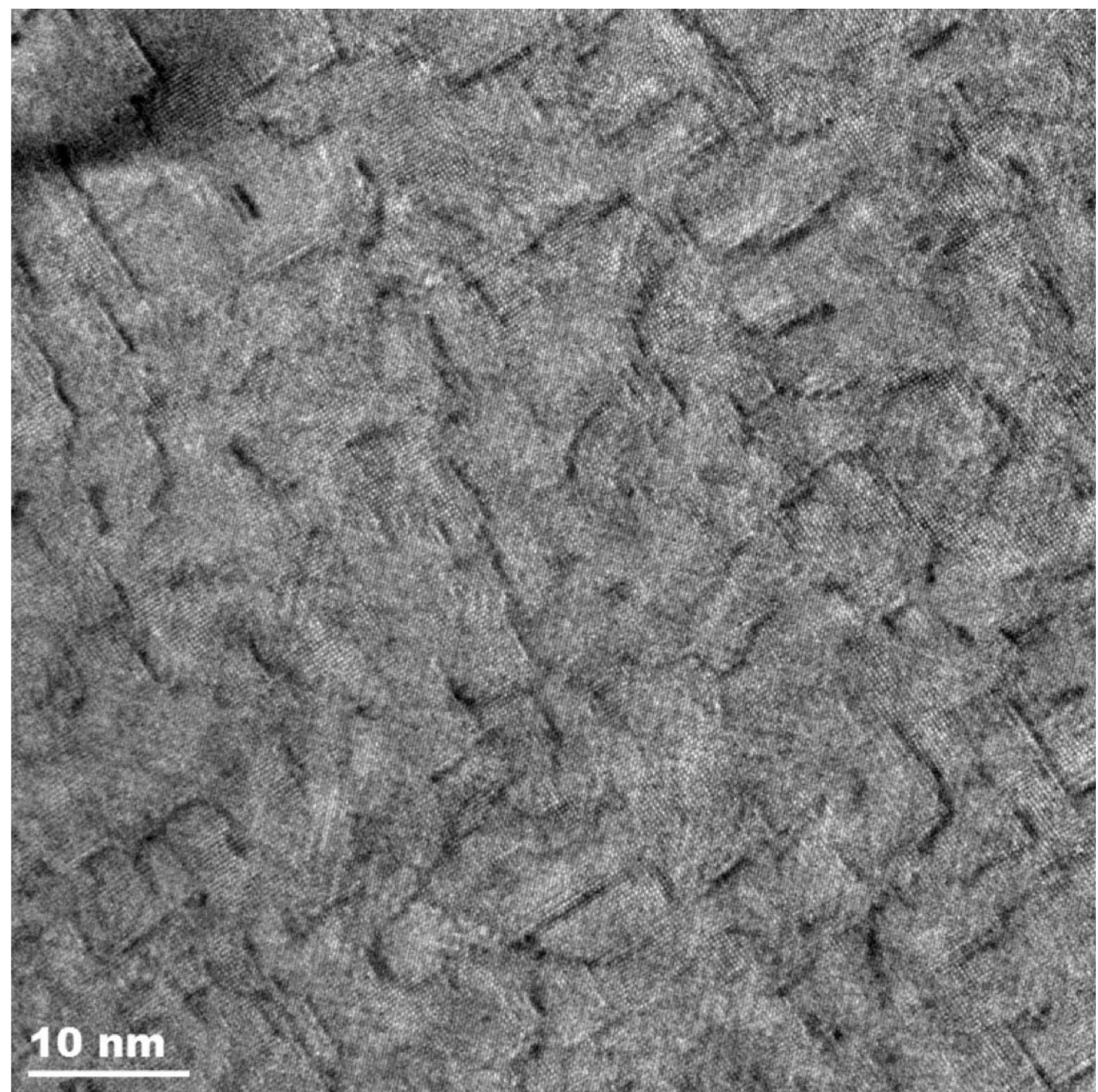

Figure 2. High resolution TEM image collected along [001] showing antiphase domain boundaries caused by a shift along $1 / 4[110]$.

\section{References}

[1] Amamou, W. et al. Physical Review Materials 2, (2018

[2] Ferreira, T. A. S. et al. Solid State Sciences 5, 383-392 (2003).

[3] Rudee, M. L. et al. Microscopy and Microanalysis 3, 126-129 (1997).

[4] Luysberg, M. et al. Physical Review B 80, 024111 (2009).

[5] Allen, L. J. et al. Ultramicroscopy 151, 11-22 (2015). 